Literature Reviews

\title{
Campylobacter Fetus Infection of the Aorta: A Case Report and Review of Literature
}

\author{
${ }^{1}$ Rabindra Ghimire, ${ }^{1,2}$ Carl Urban, ${ }^{2,3}$ Andy Lee, \\ ${ }^{4}$ Ashna Pokhrel, ${ }^{1,2}$ Wehbeh Wehbeh and ${ }^{1,2}$ Glenn Turett \\ ${ }^{I}$ The Dr James J Rahal Jr Division of Infectious Diseases, New York Hospital Queens, United States \\ ${ }^{2}$ Weill Cornell Medical College, Cornell University, New York United States \\ ${ }^{3}$ Department of Vascular Surgery, New York Hospital Queens, United States \\ ${ }^{4}$ Internal Medicine, Interfaith Medical Center, Brooklyn, NY, United States
}

\author{
Article history \\ Received: 26-04-2015 \\ Revised: $10-05-2015$ \\ Accepted: 20-06-2015 \\ Corresponding Author: \\ Rabindra Ghimire \\ The Dr James J Rahal Jr. \\ Division of Infectious Diseases, \\ New York Hospital Queens, \\ United States \\ Email: drrabindraghimire@gmail.com
}

\begin{abstract}
Campylobacter is a gram negative bacterium that exhibits tissue tropism. We report a case of Campylobacter fetus bacteremia associated with infra-renal abdominal aortitis. The patient was a poor surgical candidate so she was initially treated only medically. Her course was complicated by the development of a pseudoaneurysm. An endovascular stent was placed and the patient was given 4 more weeks of antibiotics. The patient continues to do well several months after stent placement. This case illustrates the success of medical treatment combined with stent placement in a patient who could not undergo surgery for an infected abdominal aortic pseudoaneurysm.
\end{abstract}

Keywords: Campylobacter Fetus, Mycotic Aneurysm, Endovascular Aortic Repair

\section{Introduction}

A 79 year old Chinese female with coronary artery disease, diabetes mellitus, hypertension, dyslipidemia and peptic ulcer disease presented to the Emergency Room (ER) complaining of fever and abdominal pain for 2-3 days. She described the pain as intermittent, mild, dull aching, non-radiating epigastric pain, associated with nausea, vomiting and low grade fever without chills. She denied diarrhea and complained of constipation for 1 week. The patient also reported chronic anginal chest pain for which she took sublingual nitroglycerine as needed. Review of systems was notable only for easy fatigue; she had no urinary complaints, cough, shortness of breath, headache, confusion or focal weakness.

The patient lived at home with her family and denied smoking, alcohol or recreational drug use. She emigrated from China about 12 years ago, had not travelled recently, had no animal contact and ate only well-cooked meat. She denied allergies to any medications and regularly took rosuvastatin, sitagliptin, hydrochlorothiazide, clopidogrel, losartan, ranolazine, nitroglycerine, esomeprazole, calcium and vitamin D supplements.

Her vital signs on arrival were T 100.4, HR 60, BP $112 / 55$, RR 16, SPO2 $99 \%$ on room air. Physical examination was remarkable only for mild epigastric tenderness on deep palpation. Her abnormal laboratory tests were: hemoglobin level $11 \mathrm{gm} \mathrm{dL}^{-1}$ (reference range, 13.5-17.5 $\mathrm{gm} \mathrm{dL}^{-1}$ ), White Blood Cell (WBC) count $11.85 \mathrm{k} /$ microlitre (reference range, 4.8-10.8k/microlitre), (neutrophils $87 \%$, lymphocytes $8 \%$, monocytes $3 \%$ ), creatinine $1.23 \mathrm{mg} \mathrm{dL}^{-1}$ (reference range, $0.50-0.90 \mathrm{mg} \mathrm{dL}^{-1}$ ), C-Reactive Protein (CRP) 18 $\mathrm{mg} \mathrm{L}^{-1}$ (reference range, $<0.49 \mathrm{mg} \mathrm{L}^{-1}$ ), rheumatoid factor $16 \mathrm{IU} \mathrm{mL}^{-1}$ (reference range, $<15 \mathrm{IU} \mathrm{mL}^{-1}$ ). Rapid plasma reagin, antinuclear antibody, anti-double stranded DNA antibody and human immunodeficiency virus antibody tests were all negative. Chest X-ray was normal. Abdominal CT scan revealed "marked soft tissue changes in the infrarenal aorta with involvement of the surrounding mesenteric vasculature". There was associated wall thickening of the aorta spanning $5 \mathrm{~cm}$ in the craniocaudal direction. Mildly prominent periaortic lymph nodes were also visualized (Fig. 1a).

The patient was discharged home from the ER with scheduled gastroenterology and rheumatology follow-up. She was called back after 3 days and admitted to the general medical service when blood cultures were reported as growing a gram negative bacterium in both aerobic bottles in 2 sets of cultures after $50.7 \mathrm{~h}$. The Infectious Diseases service was consulted and on further questioning, the patient complained of back pain after discharge from the ER but no fevers. She 
was afebrile and her physical exam was unchanged. Her WBC count was now normal at $8500 / \mathrm{cu} \mathrm{mm}$ (neutrophils $72 \%$, lymphocytes $16 \%$, monocytes $7 \%$ ). Repeat blood cultures (2 sets) were drawn. While awaiting identification and susceptibilities of the gram negative organism, IV meropenem 1 gram Q12h was administered. The organism was ultimately identified as Campylobacter fetus by the VITEK 2 system. Antibiotic susceptibilities as determined by E-test methodology were: doxycycline $\mathrm{MIC}=1 \mu \mathrm{g} \mathrm{mL} \mathrm{m}^{-1}$

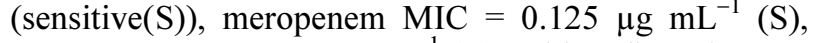
ertapenem MIC $=0.5 \mu \mathrm{g} \mathrm{mL}^{-1}(\mathrm{~S})$ and levofloxacin MIC $>32 \mu \mathrm{g} \mathrm{mL}^{-1}$ (resistant). Our working diagnosis was Campylobacter fetus bacteremia with presumed aortitis. Vascular surgery was consulted and recommended Outpatient Parenteral Antibiotic Therapy (OPAT) with follow up in the vascular clinic. The repeat blood cultures, drawn prior to starting antibiotics, were negative. The patient was discharged after 10 days with a CRP of $2.09 \mathrm{mg} \mathrm{L}^{-1}$. Treatment was continued with IV ertapenem 1 gram daily in our OPAT infusion center. In 2 weeks, her CRP normalized to $0.43 \mathrm{mg} \mathrm{L}^{-1}$. A followup CT with contrast of the abdomen and pelvis was done and revealed decreased periaortic inflammatory changes but new outpouching of contrast arising from the infrarenal aorta at the site of the previously seen arteritis, suspicious for a pseudoaneurysm (Fig. 1b). She was readmitted to the hospital and underwent an aortogram which verified the presence of a pseudoaneurysm (Fig. 2a). She was deemed a poor surgical candidate. A $9 \mathrm{~mm}$ by $59 \mathrm{~mm}$ atrium i-cast covered stent plus a $10 \mathrm{~mm}$ by $39 \mathrm{~mm}$ stent were placed endovascularly (Fig. 2b). Meropenem 1 gram Q12h treatment was continued. She developed Clostridium difficile colitis which ultimately resolved with oral metronidazole, $500 \mathrm{mg}$ every $8 \mathrm{~h}$. Her laboratory and clinical parameters remained stable. She was discharged home on hospital day 5. She continued to receive IV ertapenem in OPAT for 4 weeks beyond the date of stent placement and her CRP on completion was $0.14 \mathrm{mg} \mathrm{L}^{-1}$. Metronidazole was discontinued a week later.

The patient is followed at the ID faculty practice and is doing well several months post procedure; follow-up blood cultures remain sterile and CRP stable at 0.13 . The latest $\mathrm{CT}$ scan revealed improving inflammatory changes and decreased size of the pseudoaneurysm (Fig. 1c).

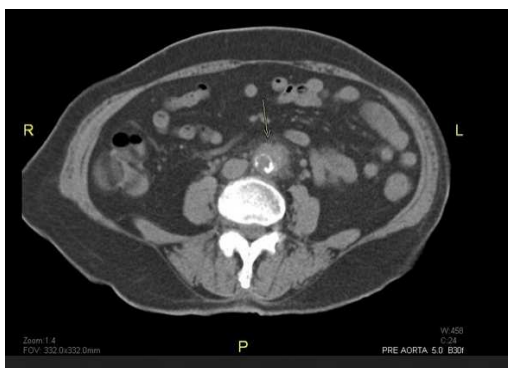

(a)

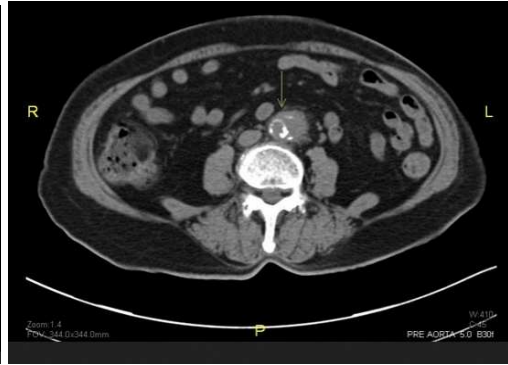

(b)

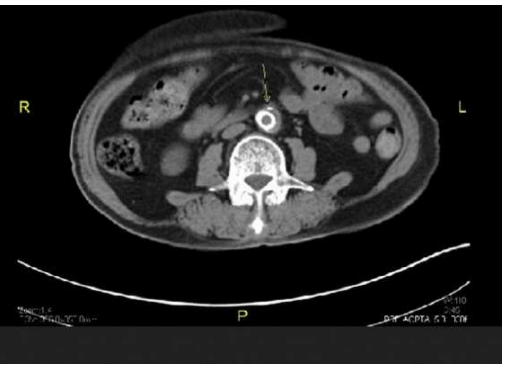

(c)

Fig. 1. Abdominal CT scan showing: 1(a) marked soft tissue changes in the infrarenal aorta with involvement of the surrounding mesenteric vasculature and associated wall thickening of the aorta (arrow), (b) decreased periaortic inflammatory changes but new outpouching of contrast arising from the infrarenal aorta at the site of the previously seen arteritis, suspicious for a pseudoaneurysm (arrow), (c) improving inflammatory changes and decreased size of the pseudoaneurysm, stent can also be visualized (arrow)

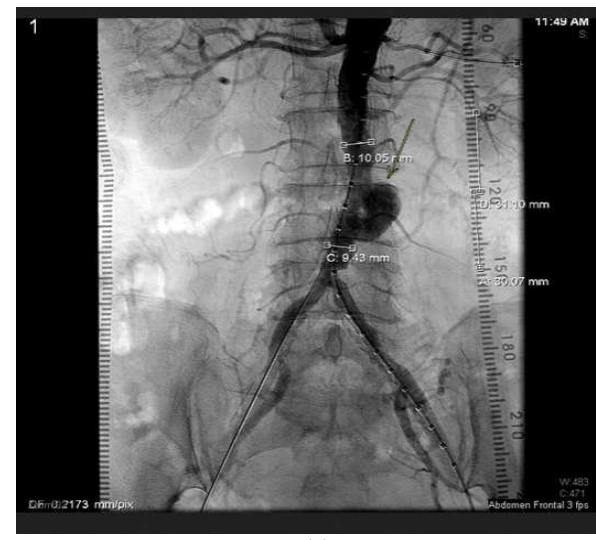

(a)

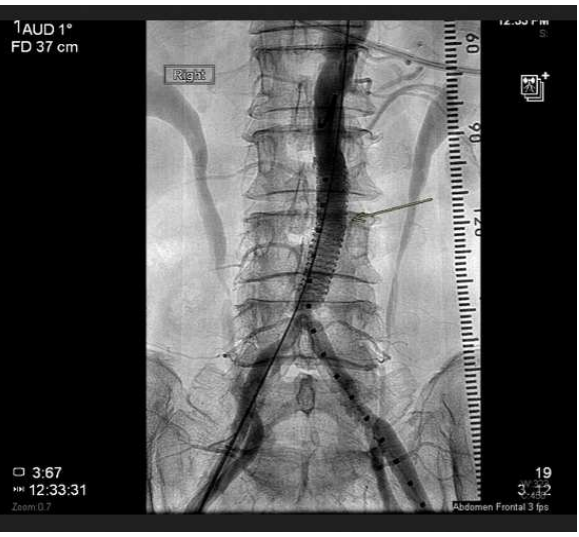

(b)

Fig. 2. (a) aortogram demonstrating a pseudoaneurysm (arrow), (b) endovascular stent (arrow) 


\section{Discussion}

Campylobacter is the Greek word for "curved rod". It is a motile, microaerophilic, non fermenting, non-spore forming, oxidase positive, gram-negative, curved bacterium. Various Campylobacter species have tissue tropism and Campylobacter fetus possesses a proteinaceous capsule-like structure (s-layer) which resists complement mediated killing and opsonization, enabling the organisms to spread to sites beyond the intestinal tract. The vascular tropism of $C$. fetus has been linked to the presence of a surface receptor with high affinity to endothelium and production of a local procoagulant that promotes thrombus formation (Morrison et al., 1990). Such tropism for vascular sites can lead to endocarditis, septic thrombophlebitis and mycotic aneurysms. Secondary seeding to the meninges, brain, bones, urinary tract, soft tissue, thyroid and prosthetic joints has also been described in the literature (Gubina et al., 1976; Wong et al., 2003; Tanaka et al., 2012; Dronda and Garcia-Arata, 1998; Yao et al., 1993; Goegebuer et al., 2007). Acquisition of C. fetus occurs via direct contact with animals, ingestion of contaminated food or water and spread by endogenous route (Taylor et al., 1979; Guerrant et al., 1978).

Campylobacter infections commonly present as inflammatory diarrhea in the normal host with fever, vomiting, abdominal cramps and watery to bloody mucoid stools. When the abdominal aorta is involved, patients often complain of back pain, as did our patient. In one series of 33 patients with mycotic aneurysms, $76 \%$ had either abdominal, back or thoracic pain, correlating with the location of the aneurysm; $48 \%$ had fever and 79\% had a leukocytosis and elevated CRP (Muller et al., 2001). Systemic infections occur among persons with generalized atherosclerosis and compromised hosts, including patients with AIDS, hypogammaglobulinemia, neoplasia, liver disease, poorly controlled diabetes mellitus, neonates and pregnant women (Pacanowski et al., 2008).

In our case, we suspect that the presence of atherosclerotic plaques in the aorta, diabetes mellitus and old age predisposed the patient to bacteremia followed by bacterial seeding of the aorta. Though bacteremic, she had a very subtle clinical presentation and remained afebrile after her initial ER discharge. Repeat blood cultures prior to initiating antibiotics were negative, suggesting she had a transient bacteremia that seeded the aorta. Although no tissue biopsy was obtained due to risk to the patient, positive blood cultures followed by the development of an aortic pseudoaneurysm support our diagnosis of presumed aortitis due to $C$. fetus.

Growth of these organisms is slow, due to low levels of metabolic activity of campylobacter in standard blood culture media. In our case, it took $50.7 \mathrm{~h}$ for growth to be detectable in culture. The median growth rate in the
BACTEC 6B aerobic and 7D anaerobic bottles for $C$. fetus is 3-5 days (Wang and Blaser, 1986). Although not routinely performed in clinical microbiology laboratories, PCR can detect and identify Campylobacter to the species level and results can be obtained on the same day (Kulkarni et al., 2002). Some authorities recommend extending the incubation of blood cultures for up to 2 weeks to enable detection of slowly growing organisms (Francioli et al., 1985). Published studies show that when biopsy cultures from the aneurysm wall and blood cultures are done, at least one is positive in 66$100 \%$ of patients (Maeda et al., 2011).

The use of Proton Pump Inhibitors (PPIs) has been associated with high rates of serious infections requiring hospitalization in veterans with decompensated cirrhosis (Bajaj et al., 2012). Proton pump inhibitors alter immunomodulatory and anti-inflammatory effects in the gut. Associations between recent use of PPIs and nontyphoidal salmonellosis and Clostridium difficile colitis have also been reported (Wu et al., 2014; Nerandzic et al., 2009). There are additional reports of increased rates of infection with enteric pathogens in persons using PPIs (Bavishi and Dupont, 2011) but no association between PPI use and campylobacter bacteremia has been demonstrated.

Due to antibiotic usage in animal food, agriculture and in humans, Campylobacter species are becoming increasingly resistant to antibiotics (Luangtongkum et al., 2009), especially fluoroquinolones and macrolides, the drugs most frequently used to treat campylobacteriosis. The Campylobacter fetus isolated in our patient was resistant to Levaquin.

During the initial course of antibacterial therapy, our patient's CRP normalized and her symptoms, including back pain improved. However, her abdominal CT scan revealed an unexpected pseudoaneurysm. This emphasizes the need for close clinical follow up in such patients, including repeat imaging studies. Our case illustrates how clinical findings and inflammatory markers may be insufficient to detect early complications. The reported risk of rupture in mycotic aneurysms is high, 50-80\% (Ting et al., 2005; Fillmore and Valentine, 2003) and if rupture occurs, mortality exceeds $75 \%$ (Blanchard 1999).

A variety of surgical techniques and treatment options have been published but most authors describe two types of procedural approaches to mycotic abdominal aortic aneurysms: open repair with a median laparotomy and Endovascular Aortic Repair (EVAR). Open repair includes resection of the infected aorta, debridement of infected tissue and in-situ reconstruction with a graft or extra-anatomic bypass, using aortoiliac or axillobifemoral procedures. Aortic resection with extraanatomic bypass carries a low risk of postsurgical infection but is associated with poor patency rates of the bypass graft despite anticoagulation and adverse outcomes including aortic stump disruption, bleeding, a higher rate of lower extremity amputation and 
compromised blood supply to the pelvis, colon and rectum (Oderich et al., 2011). Case reports have shown that in situ reconstruction with various types of grafts lead to good long-term results (Noel et al., 2002; Nevelsteen et al., 1995; Bandyk et al., 2001; Batt et al., 2003) but this has not been supported by randomized controlled trials. Weis-Muller et al. (2011) described a $42 \%$ mortality with the use of grafts and dacron patches for in-situ reconstruction of mycotic aortic aneurysm infected with various other organisms. Those grafts and the dacron patches were soaked in $600 \mathrm{mg}$ of rifampin and were covered with gauze soaked in gentamicin. He reports $30 \%$ mortality with contained rupture and $100 \%$ mortality with free rupture and no significant difference in 90-day mortality based on anatomical location of the aneurysm (Weiss-Muller et al., 2011).

Endovascular intervention is an alternative approach to open repair. Successful endovascular repair of abdominal aortic aneurysms infected with various other organisms have been reported (Corso et al., 2005). Kan et al. (2007) analyzed 48 patients with mycotic aortic aneurysms receiving endovascular treatment and reported persistent infection in $23 \%, 30$ day mortality of $10.4 \%$ due to sepsis or massive bleeding and 1 year survival of $94 \% \pm 4 \%$ in the healed group and $39 \% \pm 17 \%$ in those with persistent infection. Fever at the time of surgery and rupture of the aneurysm was predictive of persistent infection and poor outcome. Use of preoperative antibiotics for more than 1 week (OR, 0.19; 95\% CI, 0.04-1.00) and an adjunct procedure such as surgical debridement or percutaneous drainage combined with EVAR therapy (OR, 0.65; CI, 0.51-0.81) were associated with improved outcomes (Kan et al., 2007). Lai et al. (2011) describe successful treatment of group D salmonella infected infrarenal abdominal aortic aneurysms with endovascular repair without complication in two patients. Mycotic aortic aneurysms treated with endovascular stent grafts have a 30 day mortality of $36.3 \%$ if the infection persists whereas healed infections have a $2.7 \%$ mortality (Jones et al., 2005). One author documented a 38\% decrease in 30-day hospital mortality rates with EVAR when compared with open surgery, but the author suggests the possibility of selection bias (Ten Bosch et al., 2011). Hagiya et al. (2014) reviewed the literature and tabulated a total of 28 cases of mycotic abdominal aortic aneurysm caused by $C$. fetus (Hagiya et al., 2014). Of the 28 patients, 17 (60.7\%) had positive blood culture and arterial wall cultures were positive in $20(71.4 \%)$. Overall mortality was $25 \%$ (7 of 28 cases). Three patients who died before surgery, all had ruptured aortic aneurysm (File et al., 1979; Taylor et al., 1979; Allerberger et al., 1991). Two patients had EVAR and both died. One, a 76 year old male with an $11 \mathrm{~cm}$ abdominal aortic aneurysm with contained rupture underwent endovascular stent graft placement; he died of sepsis 2 weeks after the procedure (Cochennec et al., 2008). The other, a 76 year old man with an abdominal aortic aneurysm without rupture had EVAR with a dacron stent graft placed but he died 15 days postoperatively due to sepsis (Brossier et al., 2012). The other 2 fatalities occurred in a 64 year male with a ruptured aortic aneurysm who underwent aneurysm excision and axillofemoral bypass but died 7 days postoperatively (Jacobs et al., 1989) and a 68 year old male with a ruptured aortic aneurysm who had in situ graft placement but died 6 days postoperatively (Dolev et al., 1971).

There are no clear antibiotics guidelines in the literature for the treatment of aortitis and abdominal mycotic aortic aneurysms. Although surgery or EVAR is the mainstay of treatment, antimicrobial therapy remains a critical component. Regarding C. fetus most authors report treatment ranging from as short as 7 days to as long as 52 weeks. Cochennec et al. (2008) in their five cases described the use of IV amoxicillin and clavulanate or Imipenem, alone or in combination with gentamicin until the fever subsided or inflammatory markers were near normal. They followed this with long term oral antibiotics (duration not specified). Brossier et al. (2012) described five cases in which they used IV antibiotics for 30 days followed by oral antibiotics for 52 weeks but did not mention the antibiotics used. Maeda et al. (2011) reported three cases and used IV antibiotics for only 2-4 weeks until the WBC count and CRP were normal; all patients were alive at 1 year. Hagiya et al. (2014) used IV ceftriaxone 2 gram daily and ciprofloxacin $300 \mathrm{mg}$ Q12h for 36 days followed by long term oral ciprofloxacin but did not describe the dose and duration of ciprofloxacin in their patient. There is no consensus in the literature on antibiotic choice, duration or need for suppressive treatment in patients with $C$. fetus abdominal aortic mycotic aneurysms. As fluoroquinolone resistance has been reported to be as high as $32 \%$ (Pacanowski et al., 2008), using fluoroquinolones alone as initial empiric therapy could be risky. Use of a 3 rd or a 4th generation cephalosporin, an aminoglycoside and/or a carbapenem as empiric therapy while awaiting the culture and susceptibility data would seem a better approach; local antibiograms should also be taken into account.

We initially intended to treat our patient for an endovascular infection with IV antibiotics for 4 to 6 weeks. When her clinical course was complicated by a pseudoaneurysm, she underwent endovascular stenting. Since the stent was placed after 4 weeks of IV antibiotics, the patient was stable, repeat blood cultures were negative and CRP was normal, we thought that the aortic bed where the stent was placed was likely to be sterile. As a precaution, we decided to treat with 4 more weeks of IV antibiotics post-procedure. Our experience and those of others, suggests that EVAR and prolonged antibiotics is feasible when blood cultures have sterilized and the patient is clinically stable (Ting et al., 2004; Kan et al., 2007). 


\section{Conclusion}

Our case is the third report of a patient with an abdominal aortic aneurysm infected with $C$. fetus treated with endovascular stent placement and parenteral antibiotics and is the first case who survived beyond 15 days after EVAR. Our patient continues to do well 8 months later. Although aortic resection and bypass is considered to be the standard treatment for infected aortitis, we opted for EVAR because of our patient's advanced age and medical comorbidities. Prompt use of active IV antibiotics, continued through the procedure and 4 weeks beyond and short hospital stays may have led to a better outcome in our patient than those previously reported. Our experience suggests that when surgery is not deemed safe, prolonged antibiotics and EVAR is a reasonable alternative mode of treatment for mycotic abdominal aortic aneurysm due to $C$. fetus.

\section{Author's Contributions}

All the authors have contributed significantly to the preparation and writing of this manuscript.

Carl Urban: Participated in all experiments, coordinated the data-analysis and contributed to the writing of the manuscript.

Andy Lee: Coordinated the data-analysis and contributed to the surgical reviews of the manuscript.

Ashna Pokhrel: Coordinated the data-analysisand contributed to the writing of the manuscript.

Wehbeh Wehbeh: Coordinated the data-analysisand contributed to the writing of the manuscript.

Glenn Turett: Participated in all experiments, coordinated the data-analysis and contributed to the writing of the manuscript.

\section{Conflict of Interest}

The Authors declare that they have no conflict of interest.

\section{References}

Allerberger, F., M.J. Kasten and J.P. Anhalt, 1991. Campylobacter fetus subspecies fetus infection. Klin Wochenschr, 69: 813-816. DOI: 10.1007/BF01744276

Bajaj, J.S., S.M. Ratliff, D.M. Heuman and K.L. Lapane, 2012. Proton pump inhibitors are associated with a high rate of serious infections in veterans with decompensated cirrhosis. Aliment Pharmacol. Ther., 36: 866-74. DOI: 10.1111/apt.12045

Bandyk, D.F., M.L. Novotney, M.R. Back, B.L. Johnson and D.C. Schmacht, 2001. Expanded application of in situ replacement for prosthetic graft infection. J. Vasc Surg., 34: 411-19. DOI: 10.1067/mva.2001.117147
Batt, M., J.L. Magne, P. Alric, A. Muzj and C. Ruotolo et al., 2003. In situ revascularization with silver coated polyester grafts to treat aortic infection: early and midterm results. J. Vasc Surg., 38: 983-89. DOI: $10.1016 / \mathrm{S} 0741-5214(03) 00554-8$

Bavishi, C.1. and H.L. Dupont, 2011. Systematic review: The use of proton pump inhibitors and increased susceptibility to enteric infection. Aliment Pharmacol. Ther., 34: 1269-81. DOI: $10.1111 / \mathrm{j} .1365-2036.2011 .04874 . \mathrm{X}$

Blanchard, J.F., 1999. Epidemiology of abdominal aortic aneurysms. Epidemiol Rev., 21: 207-21.

Brossier, J., P. Lesprit, J. Marzelle, E. Allaire and J.P. Becquemin et al., 2012. New bacteriological patterns in primary infected Aorto-iliac aneurysms: A single-centre experience. Eur J. Vasc Surg., 40: 582-588. DOI: 10.1016/j.ejvs.2010.07.020

Cochennec, F., G. Laure, L. Philippe, D. Pascal and A. Eric et al., 2008. Aortoiliac aneurysms infected by Campylobacter fetus. J Vasc Surg., 48: 815-20. DOI: $10.1016 /$ j.jvs.2008.05.076

Corso, J.E., K. Kasirajan and R. Milner, 2005. Endovascular management of ruptured, mycotic abdominal aortic aneurysms. Am. Surg., 71: 515-17.

Dolev, E, Altmann G and B. Padeh, 1971. Vibrio fetus septicemia, a case report. Isr J. Med. Sci., 7: 1188-1191.

Dronda, F. and I. Garcia-Arata, 1998. Nevus E and De Rafael L. meningitis in adults due to C. fetus subspecies fetus. Clin. Inf. Dis., 27: 906-907.

File, M.T., J. Barnishan and J.R. Fass, 1979. Campylobacter fetus sepsis with Mycotic Aortic Aneurysm. Arch Pathol. Lab Med., 103: 143-145.

Fillmore, A.J. and R.J. Valentine, 2003. Surgical Mortality in patients with infected Aortic aneurysms. J. Am. Coll. Surgery, 196: 435-41. DOI: $10.1016 / \mathrm{S} 1072-7515(02) 01607-1$

Francioli, P., J. Herzstein, J.P. Grob, J.J. Vallotton and G. Mombelli et al., 1985. C. fetus subspecies fetus bacteremia. Arch Intern Med., 145: 289-92.

Goegebuer, T., J.P. Verhaeghe, A. Verlinde, E. De Laere and I. Surmont, 2007. Infection of the thyroid gland caused by Campylobacter fetus subsp. fetus. Acta Clin. Belg., 62: 130-133. DOI: 10.1179/acb.2007.023

Gubina, M., J. Zajc-satler, J. Mehle, B. Drinovec and F. Pikelj et al., 1976. Septicemia and meningitis with Campylobacter fetus subspecies intestinalis. Infection, 4: 115-118. DOI: 10.1007/BF01638729

Guerrant, R.L., R.G. Lahita, W.C. Winn and R.B. Roberts, 1978. Campylobacteriosis in man: Pathogenic mechanisms and review of 91 bloodstream infections. Am. J. Med., 65: 584-92. DOI: 10.1016/0002-9343(78)90845-8 
Hagiya, H., M. Matsumoto, H. Furukawa, T. Murase and F. Otsuka, 2014. Mycotic abdominal aortic aneurysm caused by Campylobacter fetus: A case report and literature review. Ann Vasc Surg., 28: 1933.e7-1933.e14.

Jacobs, J., L.J. Van, A. Navelsteen, et al., 1989. Campylobacter fetus subspecies fetus infection of an abdominal aortic aneurysm. Acta Clin. Belg., 44: $123-128$.

Jones, K.G., R.E. Bell, T. Sabharwal, M. Aukett and J.F. Reidy et al., 2005. Treatment of mycotic aortic aneurysms with endoluminal grafts. Eur J. Endovascular Surg., 29: 139-44. DOI: $10.1016 /$ j.ejvs.2004.11.008

Kan, C.D., H.L. Lee and Y.J. Yang, 2007. Outcome after endovascular stent graft treatment for mycotic aortic aneurysm: A systematic review. J Vasc Surg., 46: 906-12. DOI: 10.1016/j.jvs.2007.07.025

Kulkarni, S.P., S. Lever, J.M.J. Logan, A.J. Lawson and J. Stanley et al., 2002. Detection of Campylobacter species: A comparison of culture and polymerase chain reaction based methods. J. Clinic Path, 55: 749-753. DOI: $10.1136 /$ jcp.55.10.749

Lai, C.H., C.Y. Luo, P.Y. Lin, C.D. Kan and R.S. Chang et al., 2011. Surgical consideration of in-situ prosthetic replacement for primary infected abdominal aortic aneurysms. Eur. J. Vasc Endovasc Surg., 42: 617-624. DOI: $10.1016 /$ j.ejvs.2011.07.005

Luangtongkum, T.1., B. Jeon, J. Han, P. Plummer and C.M. Logue et al., 2009. Antibiotic resistance in Campylobacter: Emergence, transmission and persistence. Future Microbiol., 4: 189-200.

DOI: $10.2217 / 17460913.4 .2 .189$

Maeda, H., H. Umezawa, M. Goshima, T. Hattori and T. Nakamura et al., 2011. Primary infected abdominal aortic aneurysm: surgical procedures, early mortality rates and a survey of the prevalence of infectious organisms over a 30-year period. Surg Today, 41: 346-351. DOI: $10.1007 / \mathrm{s} 00595-010-4279-\mathrm{z}$

Morrison, V.A., B.K. Lloyd, J.K. Chia and C.U. Tuazon, 1990. Cardiovascular and bacteremic manifestation of C. fetus infection: Case report and review. Rev Infect Dis., 12: 387-92. DOI: 10.1093/clinids/12.3.387

Muller, B.T., O.R. Wegener, K. Grabitz, M. Pillny and L. Thomas et al., 2001. Mycotic Aneurysms of the thoracic and abdominal aorta and iliac arteries: experience with anatomic and extra-anatomic repair in 33 cases. J. Vasc Surg., 33: 106-13. DOI: $10.1067 / \mathrm{mva} .2001 .110356$

Nerandzic, M.M., M.J. Pultz and C.J. Donskey, 2009. Examination of potential mechanisms to explain the association between proton pump inhibitors and Clostridium difficile infection. Antimicrob Agents Chemother, 53: 4133-4137.

DOI: 10.1128/AAC.00252-09
Nevelsteen, A., H. Lacroix and R. Suy, 1995. Autogenous reconstruction with the lower extremity deep veins: an alternative treatment of prosthetic infection after reconstructive surgery for aortoiliac disease. J. Vasc Surg., 22: 129-34. DOI: 10.1016/S0741-5214(95)70106-0

Noel, A.A., P. Gloviczki, K.J. Cherry, H. Safi and J. Goldstone et al., 2002. Abdominal aortic reconstruction in infected fields: early results of the united states cryopreserved aortic allograft registry. J. Vasc Surg., 35: 847-52. DOI: $10.1067 / \mathrm{mva} .2002 .123755$

Oderich, G.S., J.M. Panneton, T.C. Bower, K.J. Cherry and C.M. Rowland et al., 2011. Infected aortic aneurysms: aggressive presentation, complicated early outcome but durable results. J. Vasc Surg., 34: 900-908. DOI: 10.1067/mva.2001.118084

Pacanowski, J., V. Lalande, K. Lacombe, C. Boudraa and P. Lesprit et al., 2008. Campylobacter bacteremia: Clinical features and factors associated with fatal outcome. Clin. Inf. Dis., 47: 790-796. DOI: $10.1086 / 591530$

Tanaka, A., J. Takahashi, H. Hirabayashi, N. Ogihara and K. Mukaiyama et al., 2012. A case of pyogenic spondylodiscitis caused by campylobacter fetus for which early diagnosis by magnetic resonance imaging was difficult. Asian Spine J., 6: 274-278. DOI: 10.4184/asj.2012.6.4.274

Taylor, P.R., W.M. Weinstein and J.H. Bryner, 1979. C. fetus infection in human subjects: Association with raw milk. Am. J. Med., 66: 779-83.

Ten Bosch, J.A., P.W. Cuypers, M. van Sambeek and J.A. Teijink, 2011. Current insights in endovascular repair of ruptured abdominal aortic aneurysms. Eurointervention, 7: 852-8. DOI: $10.4244 /$ EIJV7I7A133

Ting, A.C., S.W. Cheng, P. Ho and J.T. Poon, 2004. Endovascular repair for multiple salmonella mycotic aneurysms of the thoracic aorta presenting with cardiovascular syndrome. Eur J. Cardiothoracic Surg., 26: 221-224. DOI: 10.1016/j.ejcts.2004.03.035

Ting, A.C.W., S.W.K. Cheng, P. Ho, J.T.C. Poon and J.H.L. Tsu, 2005. Surgical treatment of infected aneurysms and pseudoaneurysms of the thoracic and abdominal aorta. Am. J. Surg., 189:150-54.

DOI: 10.1016/j.amjsurg.2004.03.020

Wang, W.L. and M.J. Blaser, 1986. Detection of pathogenic Campylobacter species in blood culture systems. J. Clin Microbiol., 23: 709-714.

Weiss-Muller, B.T., C. Rascanu, A. Sagban, K. Grabitz and E. Godehardt et al., 2011. Single-center experience with open surgical treatment of 36 infected aneurysms of the thoracic, thoracoabdominal and abdominal aorta. Ann Vasc Surg., 25: 1020-1025. DOI: 10.1016/j.avsg.2011.03.009 
Wong, P.L., G. Fedder and F.G. Heilmann, 2003. A man with Campylobacter endocarditis, treatable as campylobacter fetus following identification. Ned Tijdschr Geneeskd, 147: 399-403.

Wu, H.H., Y.T. Chen, C.J. Shih, Y.T. Lee and S.C. Kuo and T.L. Chen, 2014. Association between recent use of proton pump inhibitors and nontyphoid salmonellosis: a nested case-control study. Clin. Infect. Dis., 59: 1554-58. DOI: 10.1093/cid/ciu628

Yao, J.D.C., H.M.C. $\mathrm{Ng}$ and I. Campbell, 1993. Prosthetic hip joint infection due to Campylobacter fetus. J. Clin Microbiol., 31: 3323-3324. 